\title{
Demonstration of a hybrid collisional soft-x-ray laser
}

\author{
K. A. Janulewicz, ${ }^{1}$ J. J. Rocca, ${ }^{2}$ F. Bortolotto, ${ }^{1}$ M. P. Kalachnikov,${ }^{1}$ V. N. Shlyaptsev, ${ }^{3}$ W. Sandner, ${ }^{1}$ and P. V. Nickles ${ }^{1}$ \\ ${ }^{1}$ Max Born Institute, Max-Born-Strasse 2A, D-12489 Berlin, Germany \\ ${ }^{2}$ Department of Electrical and Computer Engineering, Colorado State University, Fort Collins, Colorado 80523 \\ ${ }^{3}$ Department of Applied Science, UC Davis-Livermore, California 94550
}

(Received 1 September 2000; published 6 February 2001)

\begin{abstract}
We report on a demonstration of x-ray-ultraviolet amplification following collisional excitation in a discharge-created plasma waveguide irradiated by a picosecond optical laser pulse. A capillary discharge was used to generate a sulfur plasma column with a large concentration of $\mathrm{Ne}$-like ions and a radially concave electron density profile. The intense short laser pulse rapidly heated the electrons, producing amplification in the $3 p{ }^{1} S_{0}-3 s{ }^{1} P_{1}$ transition of Ne-like $\mathrm{S}$ at $60.8 \mathrm{~nm}$. The integrated gain-length product obtained exciting a 3 -cm-long capillary with a $0.46-\mathrm{J}$ short laser pulse is 6.8 . The beam divergence was observed to decrease as a function of plasma column length, reaching $2.5 \mathrm{mrad}$ for $30-\mathrm{mm}$-long capillaries. This hybrid laser pumping scheme could lead to a new generation of efficient tabletop soft-x-ray lasers.
\end{abstract}

DOI: 10.1103/PhysRevA.63.033803

PACS number(s): 42.55.Vc, 52.50.-b

In recent years soft-x-ray lasers (XRLs) have evolved toward more compact systems based on efficient pumping schemes. Several laser excitation schemes based on either collisional electron excitation [1-3] or plasma recombination $[4,5]$ have been investigated for the development of tabletop soft XRL's. To date, two different robust pumping methods for the excitation of compact collisional XRL's have succeeded in demonstrating a laser output energy $\geqslant 10 \mu \mathrm{J}$. One is based on the generation of a transient population inversion in a laser-created plasma $[3,6,7]$, and the other in the excitation of elongated plasma columns using fast capillary discharges [1]. In the second scheme the electromagnetic force of the discharge current causes the capillary plasma column to rapidly pinch, and its density and temperature to increase markedly, creating a large quasi-steady-state density of excited Ne-like ions [1]. Capillary discharge excitation has resulted in saturated laser amplification in Ne-like $\mathrm{Ar}$ at 46.9 $\mathrm{nm}$ [8] and $\mathrm{Ne}-$ like $\mathrm{Cl}$ at $52.9 \mathrm{~nm}$ [9]. Further developments resulted in the generation of 46.9-nm nanosecond laser pulses with an average laser pulse energy of $880 \mu \mathrm{J}$ at a repetition rate of $4 \mathrm{~Hz}$, corresponding to an average power of $3.5 \mathrm{~mW}$ [10]. In turn, the transient excitation collisional scheme, which is based in the rapid heating of a precreated plasma with an intense picosecond laser pulse, has produced picosecond $\mathrm{x}$-ray laser pulses at wavelengths as short as 7 $\mathrm{nm}$ [11]. It has reduced by nearly two orders of magnitude the energy necessary to drive laser-pumped collisional soft$\mathrm{x}$-ray lasers to saturation. The first experiment involving transient collisional excitation resulted in the demonstration of a gain coefficient of $19 \mathrm{~cm}^{-1}$ at $32.6 \mathrm{~nm}$ in Ne-like Ti [3]. Further developments resulted in the saturation of the output in Ne-like Ge and $\mathrm{Ti}[6,7]$, and more recently the demonstration of saturated lasers with a $\sim 10-\mu \mathrm{J}$ output pulse energy in Ni-like Pd $(\lambda=14.7 \mathrm{~nm})$ and $\mathrm{Ag}(\lambda=13.9 \mathrm{~nm})$ using $\sim 7 \mathrm{~J}$ of total optical laser energy $[12,13]$. In the standard version of the transient excitation scheme a combination of long and short laser pulses is made to impinge on a solid target to create and subsequently rapidly heat a plasma. First the long laser pulse (typically nanosecond duration) preforms a plasma with an optimum abundance of the lasing ions in their ground state. Subsequently, the plasma electrons are very rapidly overheated by the short laser pulse. Collisions of the heated electrons with ground-state ions create a large transient inversion between the lasing levels due to the difference in the excitation rates from the ground state to the laser upper and lower levels. This results in the generation of gain coefficients that can exceed by more than one order of magnitude those obtained in the quasi-steady-state regime. Since the time scale of these processes is much shorter than any hydrodynamic effects, the losses associated with expansion cooling are inhibited. The resulting short-lived gain requires a traveling wave pump geometry to effectively utilize the energy stored in the active medium, as clearly demonstrated in recent experiments [13]. However, transient collisional XRL's are presently plagued by refraction due to steep density gradients, and require two optical laser systems to generate the pumping pulses. These refraction losses significantly increase the excitation pulse energy, preventing these lasers from achieving their full potential.

In this paper, we report on a demonstration of a scheme for pumping transient collisional soft-x-ray lasers, that could overcome these limitations. It consists of the rapid heating of a capillary discharge plasma column with an intense ultrashort laser pulse. Electron impact excitation of Ne-like S ions in a sulfur plasma waveguide generated by a lowcurrent $(3 \mathrm{kA})$ capillary discharge heated with $0.3-0.5-\mathrm{J}$ picosecond laser pulses resulted in amplification on the $3 p{ }^{1} S_{0}-3 s{ }^{1} P_{1}$ line of Ne-like $\mathrm{S}$ at $60.8 \mathrm{~nm}$. This result constitutes, to our knowledge, the first demonstration of x-rayultraviolet amplification by collisional excitation in a laserheated capillary discharge plasma waveguide. Amplification in this line has been observed in previous experiments in a quasi-steady-state regime using excitation provided by either the prepulse technique, with $E>20 \mathrm{~J}$ in the main driving laser pulse, or with a 35-37-kA current pulse produced by a high-power capillary discharge. In the first case amplification with $g=1.5 \mathrm{~cm}^{-1}$ was observed for main pulse energies as low as $20 \mathrm{~J}$, with $0.4 \mathrm{~J}$ of prepulse. However, the best results $(g l \sim 5.5)$ were obtained with a pump energy of $70 \mathrm{~J}$ in the prepulse and $\sim 400 \mathrm{~J}$ in the main pulse [14]. In case of the 
capillary discharge laser sulfur vapor was produced by ablation of the wall of an auxiliary solid sulfur capillary, and was injected into the main capillary channel, where it was subsequently excited by the fast current pulse. A gain coefficient of $0.45 \mathrm{~cm}^{-1}$ was measured in a $16-\mathrm{cm}$-long capillary [15]. In the present experiment a much smaller capillary discharge unit $\left(9 \times 34 \times 42 \mathrm{~cm}^{3}\right)$ was used as a simple, and inexpensive, source of preplasma containing a large density of Ne-like ions in the ground state. Thus, the small discharge replaces the long-pulse laser system used to preform the plasma in standard transient inversion lasers. Moreover, the discharge generates a plasma column with a concave electron density profile in the radial direction [16], that ensures a guiding of the pumping laser pulse and the amplified soft-x-ray pulse along the capillary axis. Thus in this axial pumping geometry traveling wave geometry is implemented inherently. It is worth noting that the demonstration of efficient soft-x-ray lasers has been cited as one of the main motivations for the development of plasma waveguides. Light pipes for intense laser radiation were successfully produced either in a freespace gas by focused long laser pulses [17], and in microcapillaries either irradiated by a long laser pulse [5], or in a capillary excited by a discharge current pulse $[18,19]$. The last two methods were recently successfully used to generate cold plasmas for the amplification of soft $\mathrm{x}$-rays following three-body recombination of plasma column created by optical field ionization. Gain-length products up to $g l \sim 7$ have been reported on the 2-1 line of $\mathrm{H}$-like $\mathrm{Li}$ at $13.5 \mathrm{~nm}$ [20], and a gain-length product $g l \sim 5$ was observed in the 3-2 line of H-like B at $26.2 \mathrm{~nm}$ [21]. In contrast to the cold preplasmas necessary to achieve amplification in recombination lasers, the preformed plasmas required for collisionally excited transient lasers must have a relatively high temperature to reach the Ne-like (or Ni-like) ionization stage. Recently, femtosecond laser irradiation of preformed plasma in a Mo capillary tube resulted in a strong emission increase in Nilike Mo at $18.9 \mathrm{~nm}$ [22]. In our experiment plasma columns containing a large density of Ne-like S were generated by a relatively fast discharge current pulse (100-ns rise time, 250 -ns first half-cycle duration) through an evacuated channel drilled in a sulfur rod. The rod was manufactured pressing sulfur powder at $10 \mathrm{GPa}$. Capillary channels with lengths between 10 and $30 \mathrm{~mm}$ and a diameter of 0.5 or $1.0 \mathrm{~mm}$ were investigated. The larger diameter capillaries gave no gain, and the results reported below correspond to the smaller diameter capillaries.

A simplified diagram of the experimental arrangement is shown in Fig. 1. The design of the capillary discharge resembles those previously utilized to generate plasmas by discharge ablation of evacuated channels for recombination laser amplification studies [23,24]. The preplasma was generated by discharging through the capillary channel a $0.08-\mu \mathrm{F}$ capacitor charged to energies between 0.1 and $2.0 \mathrm{~J}$. The discharge current pulse was monitored using a Rogowski coil. Maximum amplification was observed exciting plasmas generated with a peak discharge current of 2-3 $\mathrm{kA}$. After a selected delay the capillary discharge plasma was rapidly heated utilizing laser pulses of $0.3-0.5-\mathrm{J}$ energy and $\sim 1$-ps duration produced by a $\lambda=1.05 \mu \mathrm{m} \mathrm{Nd}$ :glass laser.
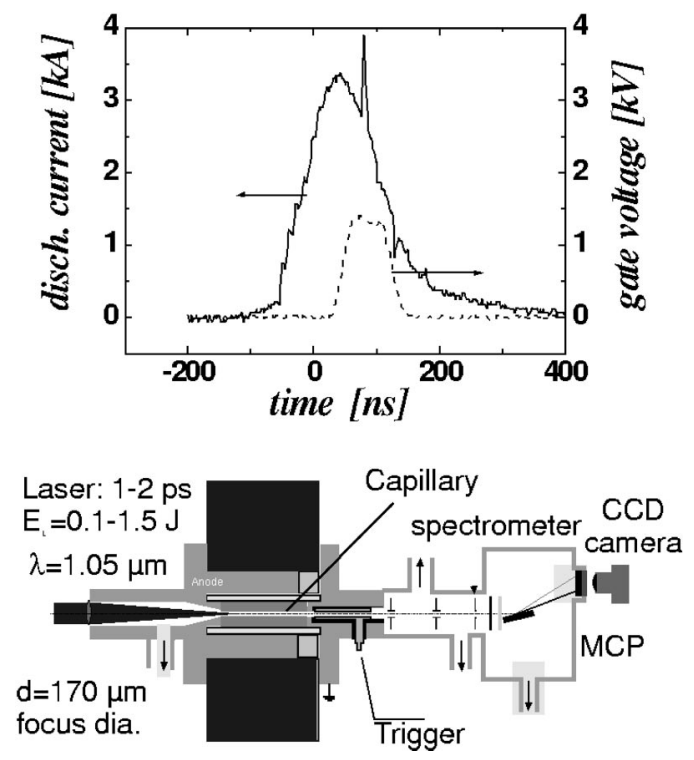

FIG. 1. Experimental setup for the spectrum measurements. The insets show temporal structure of the current wave form with the overlapped laser spike from a photodiode (solid line), and the electric signal driving the MCP (dots).

The pump laser pulses were focused onto a spot of $\sim 170-\mu \mathrm{m}$ diameter at the entrance of the capillary using a $3-\mathrm{m}$ focal length lens. The soft-x-ray radiation emitted by the plasma along the axial direction was monitored using a flatfield spectrograph having a variable space (Harada type) grating with a nominal line density of $12001 / \mathrm{mm}$. The grating, which was $\mathrm{Al}$ coated, was positioned at an angle of $3^{\circ}$ relative to the capillary axis. No filters were used. The detector consisted of a two-stage microchannel plate (MCP) intensifier, a phosphor screen and a 16-bit CCD camera. The spectrograph was calibrated using a low-pressure dc discharge in helium, and verified using OIV $(55.4 \mathrm{~nm})$ and OVI $(49.9$ and $52.0 \mathrm{~nm})$ lines from ions excited by a discharge through an evacuated polyacetal capillary. The spectral resolution of the instrument was $0.1 \mathrm{~nm}$. Gating of the MCP allowed a spectra acquisition with a temporal resolution of 40 ns. The timing of the current pulse, the laser pulse, and the MCP gate pulse were recorded for every shot using a fast digitizing oscilloscope (Tektronix TDS-3052). A more detailed description of the experimental layout was presented in Ref. [25]. The inset in Fig. 1 shows the relative time delay between these signals for a shot in which large amplification was observed. The pump laser is observed to be delayed 34 ns relative to the peak of the current pulse. Figures 2(a) and 2(b) show two spectra corresponding to discharges in a 30$\mathrm{mm}$-long capillary without and with the irradiating laser pulse present, respectively. The spectrum of Fig. 2(a), that was obtained without the pump laser, is dominated by the lines characteristic of the ionization stages SVI and SVII. This indicates that the electron temperature created by the discharge alone is sufficiently high to generate the required ground-state Ne-like (SVII) ions. However, no line emission is observed at $60.8 \mathrm{~nm}$, the wavelength of the $J=0-1$ line of Ne-like S. Strong emission on the laser line was only observed when both the discharge and the pump laser were 
a)

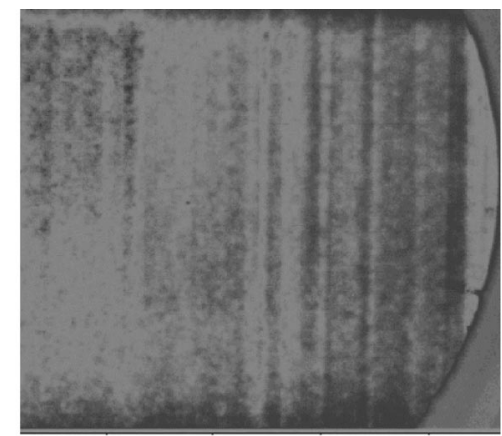

b)

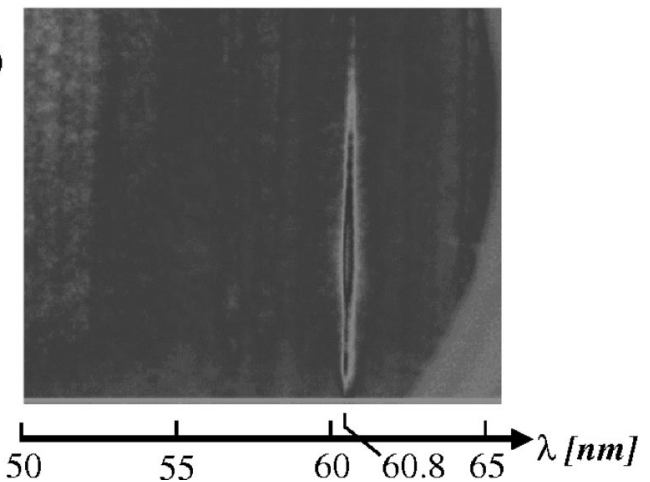

FIG. 2. (a) Spectrum of the capillary discharge alone. (b) Spectrum of the capillary discharge irradiated with a picosecond laser pulse $\left(E_{L}=320 \mathrm{~mJ}\right)$. The intensities of lines, the result of vertically integrating spectra (a) and (b), are displayed in Fig. 3(a).

present. Moreover, it was found that strong emission is possible only when the pump laser pulse delay is between 25 and $60 \mathrm{~ns}$, with the best results for $\sim 35$-ns delay. In contrast to the spectra in Fig. 2(a), the spectra obtained with both a discharge pulse and $320 \mathrm{~mJ}$ of laser excitation [Fig. 2(b)], shows very strong line emission at $60.8 \mathrm{~nm}$. This laser line clearly dominates the spectra over a $15-\mathrm{nm}$ region. Moreover, it is important to note that since the spectra were recorded with a 40-ns gate pulse to the detector, the long-lived nonlasing lines appear enhanced by two or more orders of magnitude respect to the laser line, which emission can be expected to be markedly shorter than $1 \mathrm{~ns}$. The complete dominance of the intensity of the 60.8 -nm line over other Ne-like S-ion lines that in the absence of amplification should have similar intensities (such as the $3 d^{1} P_{0}-3 p P_{1}$ line at $60.1 \mathrm{~nm}$ of SVII), but that are not observed in spectra, is strong evidence of large amplification. Additional evidence of lasing results from the significantly reduced divergence of the 60.8-nm line and its relative displacement on the detector respect to other lines, which is shown in Fig. 2(a). The divergence of the $60.8-\mathrm{nm}$ radiation was measured to be $2.5 \mathrm{mrad}$ for $30-\mathrm{mm}$-long capillaries, and $4.5 \mathrm{mrad}$ in shots corresponding to 20 -mm-long capillaries.

Figure 3 compares the cross sections of a spectra for the 3-cm-long plasma shown in Fig. 2(a) with that from the $1-\mathrm{cm}$-capillary. Measurements of the output intensity variation as a function of capillary length are presented in Fig. 4. The relative intensities shown (obtained taken into account the variation of the MCP gain with applied voltage) correspond to the averaged result obtained for each of the lengths
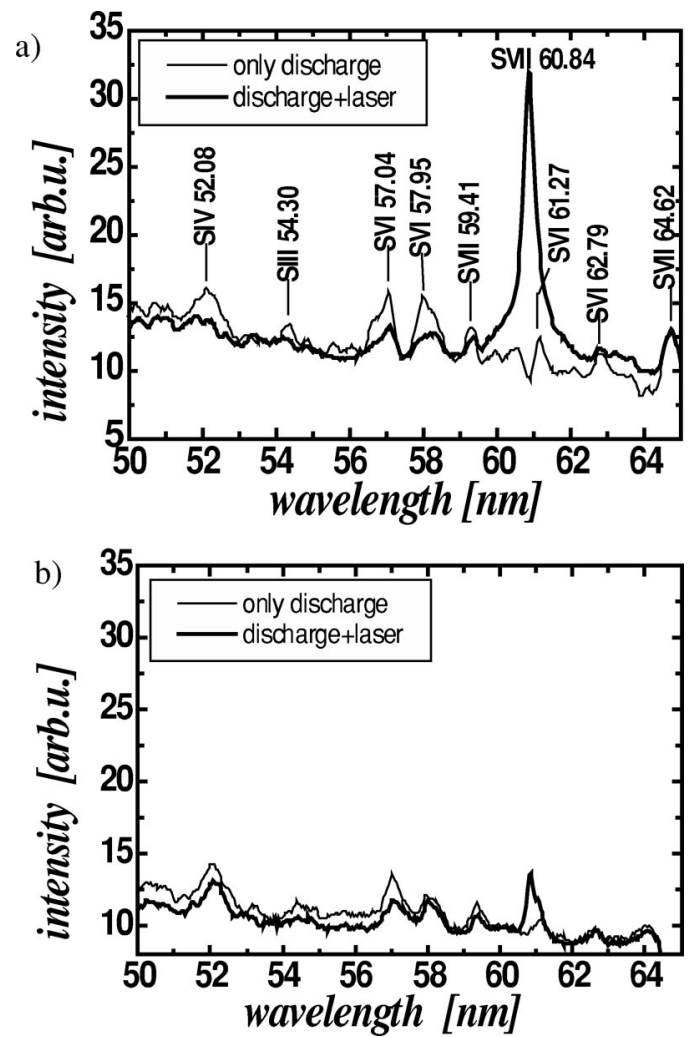

FIG. 3. Cross sections of the spectra from capillaries of different lengths: (a) $30 \mathrm{~mm}$ and (b) $10 \mathrm{~mm}$.

investigated when capillary plasma was irradiated with laser energies between 300 and $500 \mathrm{~mJ}$. The large increase in intensity of the laser line with capillary length is in contrast with the minimal changes of the intensity of the other SVII $(59.4$ and $60.1 \mathrm{~nm})$ spectral lines. While the observed supralinear increase of the laser line with plasma column length (Fig. 4) constitutes clear additional evidence of large amplification, a quantitative determination of the gain coefficient is more complicated. This difficulty is in part a consequence

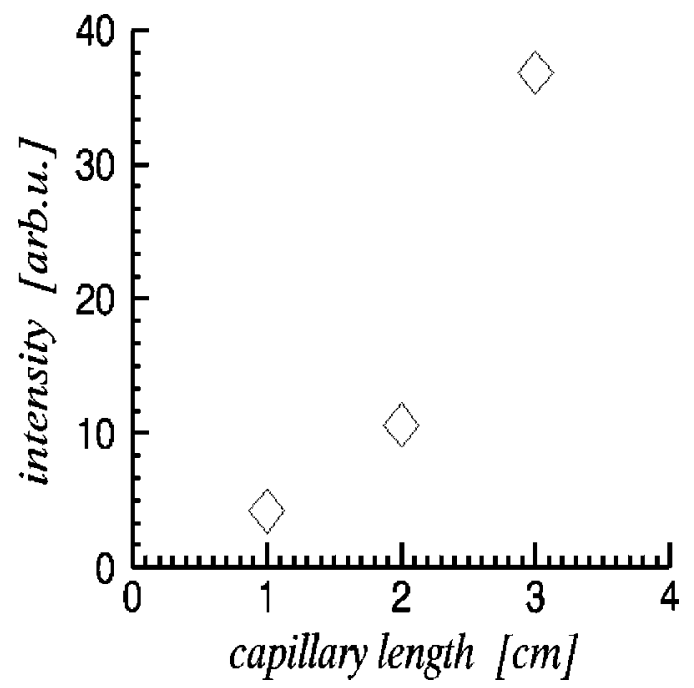

FIG. 4. Dependence of the $3 p-3 s$ (SVII, $J=0-1, \lambda$ $=60.84 \mathrm{~nm}$ ) line intensity on the capillary length 
of the axial pumping geometry, that leads to a nonuniform plasma heating in the axial direction as a result of the nonuniform absorption of the pump laser radiation, and to significant shot-to-shot variations probably caused by either noticeable jitter of the pump laser pointing or the very critical alignment of the capillaries. The short laser pump pulse is strongly absorbed during the propagation along the capillary due to the inverse bremsstrahlung. While the transmission of the pump beam through an empty capillary was between $60 \%$ and $80 \%$, the discharge caused the transmission after the current peak to be reduced strongly, depending on the delay. For time delays larger than about $80 \mathrm{~ns}$ with respect to the peak of the current pulse, the pump radiation was completely absorbed. It is interesting to note that the transmission initially increases, probably due to guiding effects caused by the wall plasma, and then decreases rapidly due to strong collisional absorption. Approximately $30 \mathrm{~ns}$ after the current peak there is a small and a short-lasting increase of the transmissivity, that corresponds well with the observed optimal delay for lasing. This effect, which was observed for capillaries of different lengths, may be ascribed to the fact that at this time an increase in the optical guiding is changing the beam divergence. Nevertheless, this increase in the transmission does not alter the fact that the active medium is nonuniformly pumped in the axial direction, with a reduction in the energy deposition at the end of the longer capillaries. The reduction of the pump intensity as a function of plasma length causes a significant drop of the electron temperature along the axis. Applying the analytical formulas for the inverse bremsstrahlung rate in a low-temperature plasma irradiated with a high-intensity radiation, derived in Ref. [26], to our initial plasma conditions $\left(n_{e}=2 \times 10^{19}\right.$ and $\left.T_{e}=20 \mathrm{eV}\right)$, it is possible to estimate that a decrease of the incident intensity from $10^{15}$ to $10^{14} \mathrm{~W} / \mathrm{cm}^{2}$ corresponds to a reduction in the electron temperature from about 174 to $75 \mathrm{eV}$. In turn, this decrease of the plasma temperature along the capillary axis results in a reduction of the gain coefficient. Based on the above considerations the standard technique for determining the gain assuming a constant gain coefficient does not apply here.

For sufficiently large plasma column lengths this problem of a decreasing gain coefficient with plasma column length will be faced by all longitudinally pumped schemes relying on the radiation absorption. Nevertheless, an estimate of the gain can be obtained by locally fitting the experimental data with a variable gain coefficient. The effective $g l$ product can be obtained by integrating the gain coefficient over the plasma column length. This technique, which was recently analyzed in Ref. [27], was previously used by Dunn et al. [28] in estimating the gain of non-traveling-wave transient lasers in which the gain coefficient decreases as a function of plasma column length. In our case a gain coefficient of 4.6 $\mathrm{cm}^{-1}$ has been obtained for the first 1-cm-long section of the plasma using an arbitrarily chosen small value of the detectable signal at the origin. The gain coefficient decreases to less than $1.2 \mathrm{~cm}^{-1}$ in the last two centimeter of the plasma. Part of the gain decrease in the case of longer capillary can be expected to be the result of the laser pump energy depletion with the capillary length, as explained above. Hence an effective gain coefficient for the $3-\mathrm{cm}$ capillary is about 2.3 $\mathrm{cm}^{-1}$, and a gain-length product of $g l=6.8$ has been achieved with only $0.46 \mathrm{~J}$ of laser pump energy and $2.2 \mathrm{~J}$ of total pump energy $\left(E_{P}\right)$, taking into account the energy dissipated by the discharge. Considering the frequently quoted $g l / E_{P}$ ratio, commonly used as a rough estimate of the pump scheme efficiency, we obtain a value of $3.1 \mathrm{~J}^{-1}$, one of the highest values of this parameter reported to date for collisionally pumped ultrashort wavelength lasers. While we have no direct proof that the laser action observed occurs in the transient regime, there are reasons to expect that at least part of the amplification occurs in this regime. The rapid heating of the electrons by the picosecond laser pulse to temperatures in excess of $150 \mathrm{eV}$ should produce a large transient inversion, at least in the first fraction of the capillary. In any case, the gain coefficient obtained is significantly larger than the $0.45 \mathrm{~cm}^{-1}$ previously observed in this line under discharge excitation alone [15], a situation much closer to quasi-steady-state, and also exceeds that obtained with the excitation energy of nearly $21 \mathrm{~J}\left(1.5 \mathrm{~cm}^{-1}\right)$ applying the prepulse technique to a flat sulfur target [14].

In conclusion, we have demonstrated x-ray-ultraviolet amplification following collisional electron excitation of a capillary discharge plasma. This hybrid soft-X-ray pumping scheme combines the advantages of efficient preplasma generation and a concave electron-density profile of a capillary discharge with the increased gains that are possible following rapid heating with a picosecond laser pulse. The resulting enhanced pump laser energy deposition efficiency and reduced refraction observed in this scheme offer significant potential in scaling collisionally pumped tabletop soft-x-ray lasers to shorter wavelengths.

The authors thank Professor Geoff Pert of the University of York for helpful discussions, and Dana Burt from Colorado State University for his assistance with the capillary discharge. J.J.R. gratefully acknowledges the support of the W. M. Keck Foundation. This work has been supported by the DFG Project No. SPP 1053 "Fundamentals of new pumping methods for X-ray lasers," and by the U.S. National Science Foundation.
[1] J. J. Rocca, V. N. Shlyaptsev, F. G. Tomasel, O. D. Cortazar, D. Hartshorn, and J. L. A. Chilla, Phys. Rev. Lett. 73, 2192 (1994).

[2] B. E. Lemoff, G. Y. Lin, C. L. G. III, C. P. Barty, and S. E.
Harris, Phys. Rev. Lett. 74, 1574 (1995).

[3] P. V. Nickles, V. N. Shlyaptsev, M. P. Kalachnikov, M. Schnuerer, I. Will, and W. Sandner, Phys. Rev. Lett. 78, 2748 (1997). 
[4] Y. Nagata, K. Midorikawa, K. Obara, M. Tashiro, and K. Toyoda, Phys. Rev. Lett. 71, 3774 (1993).

[5] D. V. Korobkin, C. H. Nam, A. Goltsov, and S. Suckewer, Phys. Rev. Lett. 77, 5206 (1996).

[6] P. J. Warwick, C. L. S. Lewis, M. P. Kalachnikov, P. V. Nickles, M. Schnuerer, A. Behjat, A. Demir, G. J. Tallents, D. Neely, E. Wolfrum, J. Zhang, and G. J. Pert, J. Opt. Soc. Am. 15, 1808 (1998).

[7] M. P. Kalachnikov, P. V. Nickles, M. Schuerer, W. Sandner, V. N. Shlyaptsev, C. Danson, D. Neely, E. Wolfrum, J. Zhang, A. Behjat, A. Demir, G. J. Tallents, P. J. Warwick, and C. L. S. Lewis, Phys. Rev. A 57, 4778 (1998).

[8] J. J. Rocca, D. P. Clark, J. L. A. Chilla, and V. N. Shlyaptsev, Phys. Rev. Lett. 77, 1476 (1996).

[9] M. Frati, M. Seminario, and J. J. Rocca, Opt. Lett. 25, 1022 (2000).

[10] B. R. Benware, C. D. Macchietto, C. H. Moreno, and J. J. Rocca, Phys. Rev. Lett. 81, 5804 (1998).

[11] A. G. MacPhee, R. M. N. O’Rouke, C. L. S. Lewis, J. Y. Lin, A. Demir, G. J. Tallents, J. Collier, D. Neely, D. Ros, Ph. Zeitoun, S. P. McCabe, P. Simms, and G. J. Pert, Proceedings of the Sixth International Conference on X-ray Lasers, IOP Conf. Proc. No. 159 (Institute of Physics and Physical Society, London, 1999), p. 75.

[12] J. Dunn, J. Nilsen, A. L. Osterheld, Y. Li, and V. N. Shlyaptsev, Opt. Lett. 24, 101 (1999).

[13] J. Dunn, Y. Li, A. L. Osterheld, J. Nilsen, J. R. Hunter, and V. N. Shlyaptsev, Phys. Rev. Lett. 84, 4834 (2000).
[14] Y. Li, P. Lu, G. Pretzler, and E. E. Fill, Opt. Commun. 133, 196 (1997).

[15] F. G. Tomasel, J. J. Rocca, V. N. Shlyaptsev, and C. D. Macchietto, Phys. Rev. A 55, 1437 (1997).

[16] C. H. Moreno, M. C. Marconi, V. N. Shlyaptsev, and J. J. Rocca, IEEE Trans. Plasma Sci. 27, 6 (1999).

[17] H. M. Milchberg, C. G. Durfee III, and J. Lynch, J. Opt. Soc. Am. B 12, 731 (1995).

[18] A. Zigler, Y. Ehrlich, C. Cohen, J. Krall, and P. Sprangle, J. Opt. Soc. Am. B 13, 68 (1996).

[19] S. M. Hooker, D. J. Spence, and R. A. Smith, J. Opt. Soc. Am. B 17, 90 (2000).

[20] D. V. Korobkin, A. Goltsov, A. Morozov, and S. Suckewer, Phys. Rev. Lett. 81, 1607 (1998).

[21] A. Morozov, Y. Ping, S. Suckewer, A. Bartnik, and H. Fiedorowicz, Proc. SPIE 3156, 214 (1997).

[22] R. Li (private communication).

[23] M. C. Marconi and J. J. Rocca, Appl. Phys. Lett. 54, 2180 (1989).

[24] J. J. Rocca, M. C. Marconi, and F. G. Tomasel, IEEE J. Quantum Electron. 29, 182 (1993).

[25] K. A. Janulewicz, F. Bortolotto, P. J. Warwick, M. P. Kalachnikov, V. N. Shlyaptsev, W. Sandner, J. J. Rocca, and P. V. Nickles, Proc. SPIE 3776, 37 (1999).

[26] G. J. Pert, Phys. Rev. E 51, 4778 (1995).

[27] R. Tommasini and E. E. Fill, Phys. Rev. A 62, 034701 (2000).

[28] J. Dunn, A. L. Osterheld, R. Shepherd, W. E. White, V. N. Shlyaptsev, and R. E. Stewart, Phys. Rev. Lett. 80, 2825 (1998). 